\title{
An adaptive singular spectrum analysis method for extracting brain rhythms of electroencephalography
}

\author{
Hai Hu ${ }^{1}$, Shengxin Guo ${ }^{2}$, Ran Liu ${ }^{2}$, Peng Wang ${ }^{\text {Corresp. } 1}$ \\ 1 State Key Laboratory of Precision Measurement Technology and Instruments, Tsinghua University, Beijing, China \\ 2 Department of Biomedical Engineering, School of Medicine, Tsinghua University, Beijing, China \\ Corresponding Author: Peng Wang \\ Email address: peng@mail.tsinghua.edu.cn
}

Artifacts removal and rhythms extraction from electroencephalography (EEG) signals is important for portable and wearable EEG recording devices. Incorporating a novel grouping rule, we proposed an adaptive singular spectrum analysis (SSA) method for artifacts removal and rhythms extraction. Based on the EEG signal amplitude, the grouping rule determines adaptively the first one or two SSA reconstructed components as artifacts and removes them. The remaining reconstructed components are then grouped based on their peak frequencies in the Fourier transform to extract the desired rhythms. The grouping rule thus enables SSA to be adaptive to EEG signals containing different levels of artifacts and rhythms. The simulated EEG data based on the Markov Process Amplitude (MPA) EEG model and the experimental EEG data in the eyes-open and eyes-closed states were used to verify the adaptive SSA method. Results showed a better performance in artifacts removal and rhythms extraction, compared with the wavelet decomposition (WDec) and another two recently reported SSA methods. Features of the extracted alpha rhythms using adaptive SSA were calculated to distinguish between the eyes-open and eyes-closed states. Results showed a higher accuracy (95.8\%) than those of the WDec method (79.2\%) and the infinite impulse response (IIR) filtering method (83.3\%). 


\section{An Adaptive Singular Spectrum Analysis Method for Extracting}

\section{Brain Rhythms of Electroencephalography}

3 Hai Hu${ }^{1}$, Shengxin $\mathrm{Guo}^{2}$, Ran $\mathrm{Liu}^{2}$, Peng Wang ${ }^{1}$

$4{ }^{1}$ State Key Laboratory of Precision Measurement Technology and Instruments, Tsinghua University, 5 Beijing, China

$6{ }^{2}$ Department of Biomedical Engineering, School of Medicine, Tsinghua University, Beijing, China

8 Corresponding Author:

$9 \quad$ Peng Wang ${ }^{1}$

10 Department of Precision Instrument, Tsinghua University, Beijing, PR China

11 Email address: E-mail: peng@mail.tsinghua.edu.cn

12 
13 Abstract

14 Artifacts removal and rhythms extraction from electroencephalography (EEG) signals is 15 important for portable and wearable EEG recording devices. Incorporating a novel grouping rule,

16 we proposed an adaptive singular spectrum analysis (SSA) method for artifacts removal and

17 rhythms extraction. Based on the EEG signal amplitude, the grouping rule determines adaptively

18 the first one or two SSA reconstructed components as artifacts and removes them. The remaining reconstructed components are then grouped based on their peak frequencies in the Fourier transform to extract the desired rhythms. The grouping rule thus enables SSA to be adaptive to EEG signals containing different levels of artifacts and rhythms. The simulated EEG data based on the Markov Process Amplitude (MPA) EEG model and the experimental EEG data in the eyes-open and eyes-closed states were used to verify the adaptive SSA method. Results showed a better performance in artifacts removal and rhythms extraction, compared with the wavelet decomposition (WDec) and another two recently reported SSA methods. Features of the extracted alpha rhythms using adaptive SSA were calculated to distinguish between the eyesopen and eyes-closed states. Results showed a higher accuracy $(95.8 \%)$ than those of the WDec method (79.2\%) and the infinite impulse response (IIR) filtering method (83.3\%).

\section{Introduction}

Electroencephalography (EEG) is a measurable voltage resulting from electrical activity of the brain neurons (Niedermeyer \& da Silva 2005). Spontaneous EEG consists of several rhythms of

32 different frequencies: delta $(1-4 \mathrm{~Hz})$, theta $(4-8 \mathrm{~Hz})$, alpha $(8-13 \mathrm{~Hz})$, beta $(13-30 \mathrm{~Hz})$ and 
33 gamma $(>30 \mathrm{~Hz})$, each containing information about different brain activity. For example, the

34 alpha rhythm reflects attention demands, and the beta rhythm reflects emotional and cognitive

processes (Rowland et al. 1985). Because of its feasibility and convenience, EEG has been

widely studied for the brain physiological states monitoring (Jones et al. 2014; Ko et al. 2009;

$\mathrm{Ng} \&$ Chan 2005a).

Rhythms extraction from the EEG signals is important for portable and wearable EEG

recording devices, which have attracted much attention in recent studies (Gargiulo et al. 2008;

Van Bavel et al. 2008). Yu M. Chi et al. developed a system using wireless non-contact EEG

electrodes to collect EEG signals for the alpha rhythm extraction (Chi \& Cauwenberghs 2010).

Ranjit et al. utilized changes in the EEG alpha rhythm during eye closure as a switch for electrical devices used for severely impaired people (Thuraisingham et al. 2007). In addition, the

EEG alpha rhythm was also used as a measure of resting-state arousal and activation (Barry et al. 2007). However, the EEG signal is always contaminated by artifacts, including electrooculogram (EOG), electromyography (EMG), baseline drift and stochastic noise, which interfere the rhythms extraction (Azarbad et al. 2014; Daly et al. 2013; Teixeira et al. 2006).

To extract the desired rhythms accurately from the interfered EEG signals, various methods have been proposed for artifacts removal (Azarbad et al. 2014; Daly et al. 2013; Teixeira et al. 2006). In multi-channel EEG recording, visual inspection is useful for artifacts removal. However, this method is not applicable for portable or wearable devices with single-channel EEG recording (Nunez et al. 1999). Therefore, many efforts have been paid on algorithm for artifacts removal (Teixeira et al. 2006). He et al. used regression analysis (RA) and adaptive 
54 filtering (AF) techniques for EOG artifact removal (He et al. 2004). These methods required a

separately recorded EOG signal as a reference, which was however always contaminated.

Wallstrom et al. applied the independent component analysis (ICA) method to automatically remove the ocular artifacts in EEG signals. However it distorted the EEG signals in the range of 5-20 Hz (Wallstrom et al. 2004). Azami et al. used the wavelet transform (WT) method for artifacts removal. However, this method was relatively slow, and could not separate components overlapping in time-frequency space (Azami \& Sanei 2012; Azami \& Sanei 2014).

SSA is another powerful method for time series analysis. It enables separation of different sources even overlapping in time-frequency space, and has been recently applied for EEG artifacts removal and rhythms extraction. Maddirala et al. proposed a new grouping criterion in SSA to construct the reference signal for EOG artifact removal (Maddirala \& Shaik 2016b). Based on the local mobility of the eigenvectors, Maddirala et al. then proposed another grouping criterion to remove the motion artifact, which performed better than the existing methods (Maddirala \& Shaik 2016a). Mohammadi et al. proposed a SSA method with a new grouping criterion based on the eigenvalue pairs to extract the main rhythms from sleep EEG signals (Mohammadi et al. 2015; Mohammadi et al. 2016). Akar et al. proposed a wavelet decomposition-SSA based method for noise removal and desired components extraction from EEG signals. It was successfully applied for schizophrenics' brain dynamics analysis (Akar et al. 2015). In the SSA method, the grouping rule is important for SSA reconstruction. However, there is no general grouping rule. For a specific study, it depends on the research target, the types of signals and noise involved (Azami \& Sanei 2014; Kouchaki 2014; Sanei et al. 2012). 
In this paper, we proposed a novel grouping rule for SSA reconstruction to remove artifacts

76

77 and extract rhythms from EEG signals. EEG signals were processed by SSA to obtain a series of reconstructed components. The first one or two reconstructed components determined by the signal amplitude, were then regarded as artifacts and removed. Finally, the reconstructed components were grouped, based on their peak frequencies in the Fourier transform, to extract the specific EEG rhythms. Proof-of-concept experiments were performed to verify the proposed adaptive SSA method in extracting the EEG alpha rhythm and distinguishing between the eyesopen and eyes-closed states.

\section{Methods}

\subsection{Adaptive SSA method}

SSA consists of two stages: decomposition and reconstruction. Decomposition involves timedelay embedding called Takens' theorem (Takens 1981), followed by singular value decomposition (SVD) (Mees et al. 1987). Reconstruction involves grouping and diagonal averaging (Vautard et al. 1992). In the time-delay embedding step, the single-channel EEG time series $\mathbf{s}=\left(s_{1}, s_{2}, \mathrm{~K}, s_{N}\right)^{T}$, superscript $T$ denoting the transpose of a vector, is mapped onto a multidimensional trajectory matrix $\mathbf{X}$ using a sliding window.

$$
\mathbf{X}=\left(\mathbf{S}_{1}, \mathbf{S}_{2}, \mathrm{~K}, \mathbf{S}_{K}\right)=\left(\begin{array}{cccc}
s_{1} & s_{2} & \mathrm{~L} & s_{K} \\
s_{2} & s_{3} & \mathrm{~L} & s_{K+1} \\
\mathrm{M} & \mathrm{M} & \mathrm{O} & \mathrm{M} \\
s_{L} & s_{L+1} & \mathrm{~L} & s_{N}
\end{array}\right)
$$


92 where $L$ denotes the window length (or embedding dimension), $K=N-L+1$, and $\mathbf{S}_{i}(1 \leq i \leq k)$

93 denotes the lagged vector. Next, the SVD of the matrix $\mathbf{X}$ is computed as:

94

95

96

97

98

99

100

101

102

103

104

105

106

107

108

109

110

$$
\mathbf{X}=\sum_{i=1}^{L} \mathbf{X}_{i}=\sum_{i=1}^{L} \sqrt{\lambda_{i}} \mathbf{v}_{i} \mathbf{p}_{i}^{T}
$$

where $\mathbf{X}_{i}$ denotes the elementary matrice, $\lambda_{i}$ denotes the eigenvalue of covariance matrix $\mathbf{C}=\mathbf{X} \mathbf{X}^{T}$ in the decreasing order of magnitude $\left(\lambda_{1} \geq \lambda_{2} \geq L \geq \lambda_{L} \geq 0\right), \quad \mathbf{v}_{i}$ denotes the corresponding eigenvector, and $\mathbf{p}_{i}=\mathbf{X}^{T} \mathbf{v}_{i} / \sqrt{\lambda_{i}}$.

Then, the diagonal averaging step reconstructs several time series from the corresponding elementary matrices. The reconstructed time series are generally called reconstructed components (RCs). Finally, based on the proposed adaptive grouping rule, the RCs are grouped for artifacts removal and rhythms extraction.

\section{The adaptive grouping rule}

With the SSA treatment, the original EEG time series are decomposed into a set of RCs. The first several RCs dominate the trend of the EEG time series, which is represented by the large artifacts (Teixeira et al. 2006). Here, the first one or two RCs, determined by the EEG amplitude, are grouped as artifacts. When the amplitude is large, indicating a high level of artifacts, the first two RCs are grouped. Otherwise, the first RC is grouped:

$$
\text { Artifacts }= \begin{cases}R C_{1}+R C_{2} & \max (\mathbf{s})>V_{0} \\ R C_{1} & \max (\mathbf{s})<V_{0}\end{cases}
$$

where $\max (\mathbf{s})$ denotes the EEG amplitude, $V_{0}$ is a threshold. Since the amplitude of the spontaneous EEG without artifacts is usually below $100 \mu \mathrm{V}(\mathrm{Ng} \&$ Chan 2005b), the threshold 
111 was set as $V_{0}=200 \mu \mathrm{V}$.

112 After artifacts removal, the rhythms are then extracted from the EEG time series. Rhythms

113 are the oscillatory components of EEG time series, including, but not limited to, the periodic

114 components. In order to extract the EEG rhythms, the RCs are then divided into two groups: the

115 periodic and non-periodic components. Firstly, the periodic components are extracted. Generally,

116 a periodic time series will be factorised into some eigenvalue pairs with similar amplitude using

117 SSA (Vautard et al. 1992). So, RCs with similar eigenvalues belong to the same periodic

118 component. They are summed up as a periodic component (PC). The similarity of the

119 eigenvalues for periodic component extraction is determined by the following criterion:

120

$$
\left|1-\frac{\lambda_{j}}{\lambda_{i}}\right|<0.05
$$

121 Then, these PCs and the remaining non-periodic RCs are grouped. Each PC and RC will fall into

122 a narrow frequency band, when the window length $L$ is large enough (Kouchaki 2014;

123 Mohammadi et al. 2015; Sanei et al. 2011). So, the peak frequency in the Fourier transform can

124 be used to represent the frequency range of the PC and RC.

$$
f_{\max }=\underset{f}{\arg \max }\{a b s[F F T(R C)]\}
$$

where $f_{\max }$ is the peak frequency, $F F T(R C)$ is the fast Fourier transform of the RC or PC. RCs and PCs with the peak frequencies in the same rhythm band are finally clustered into the same group, which constitutes the brain rhythm.

The pseudo-code of the adaptive SSA method is shown in Fig. 1. 
Algorithm 1 Adaptive SSA Method for Alpha Rhythms Extraction

input s: single-channel EEG time series

output C: corrected EEG time series after artifacts removal

$\boldsymbol{\alpha}$ : extracted alpha rhythm

procedures Artifacts removal and rhythms extraction

(1) RCs: apply the SSA on s with the window length $L=40$ and derive a set of RCs by Eq.1-2.

(2) $\mathbf{C}$ : derive the artifacts (A) by Eq.3, and $\mathbf{C}=\mathbf{s}-\mathbf{A}$

(3) PCs: group the RCs and derive periodic components (PCs) by Eq.4

(4) $\alpha$ : group the PCs and the non- periodic RCs to derive alpha rhythm by Eq.5

130

131

132

133

134

135

136

137

return $\mathbf{C}, \boldsymbol{\alpha}$

Fig. 1 The pseudo-code of the adaptive SSA method.

Window length $L$ is selected based on the lowest frequency of interest $\left(L \geq f_{s} / f_{l}\right)$ to capture at least one period of the expected component (James \& Lowe 2003). In this paper, the window length $L$ is set to be 40 . After extracting the desired rhythms, the features of the rhythms are obtained for each EEG signal. The mean, standard deviation (SD), power and power ratio of different rhythms are usually selected as the features (Mohammadi et al. 2015). In this study, the power $\left(P=\sum V_{\alpha}^{2} / N\right)$ of the alpha rhythm were selected.

\subsection{Data source}


139 Simulated EEG data with known parameters and experimental EEG data were used to verify the

140 validity of the adaptive SSA method in artifacts removal and alpha rhythm extraction.

141 Simulated EEG data

142 Simulated EEG data consisted of two parts: the spontaneous EEG and the artifacts. The 143 spontaneous EEG has two major characteristics: rhythmic oscillations and randomness. The 144 artifacts mainly consist of EOG, baseline drift and white Gaussian noise. model developed by Nishida et al (Nishida et al. 1986). In the MPA EEG model, the EEG rhythmic oscillations were represented by sinusoidal waves, and the EEG randomness was represented by the stochastic process amplitude of the first-order Markov process (Al-Nashash et al. 2004; Bai et al. 2000; Bai et al. 2001). The spontaneous EEG was generated by a combination of $K$ different oscillations,

$$
s(n \Delta t)=\sum_{i=1}^{K} a_{i}(n \Delta t) \sin \left(2 \pi m_{i} n \Delta t+\theta_{i}\right)
$$
where $\gamma$ is the coefficient of the first-order Markov process, $\xi$ is a random increment of Gaussian where $n$ is the sample number, $\Delta t$ is the time interval, $K$ is the number of rhythms, $m$ is the dominant frequency, $\theta$ is an arbitrary value representing the initial phase, $a$ is the rhythmic amplitude obtained from a first-order Gauss-Markov process,

$$
a_{i}[(n+1) \Delta t]=\gamma_{i} a_{i}(n \Delta t)+\xi_{i}(n \Delta t)
$$

157 distribution with zero mean and variance $\sigma$. 

parameters of the MPA EEG model were determined in the frequency domain (shown in Table

Fig. 2B. Finally, the spontaneous EEG was generated by a combination of the four rhythms, as

The detailed mathematical description of the spontaneous EEG simulation is shown in the

A)

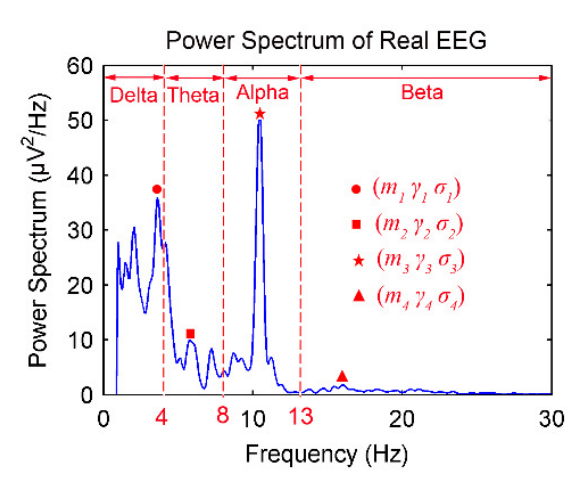

B)

$$
\{
$$

168

169

170

171

172

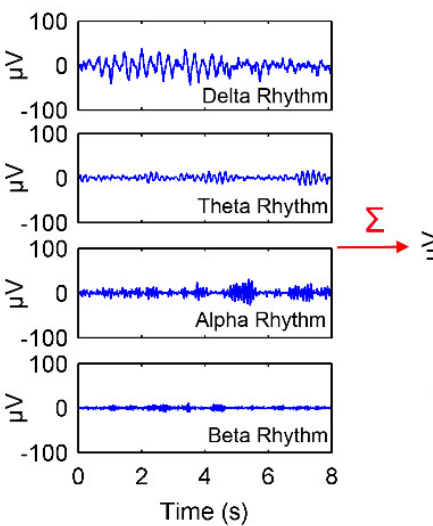

C)

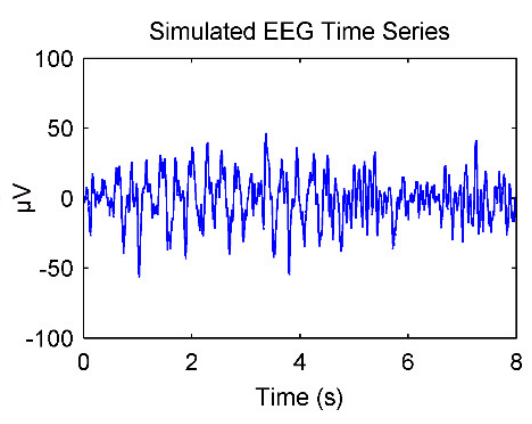

Fig. 2 Procedures of the spontaneous EEG simulation based on the MPA EEG model. (A) The power spectrum of a real EEG. Parameters of the model were determined based on the power spectrum. (B) The generated four rhythms: delta, theta, alpha and beta. (C) The simulated spontaneous EEG generated by a combination of the four rhythms. 
174 artifact and is caused by eye blinks and ocular movement. It is characterized by large amplitude,

175 low-frequency electro-potential shift. Baseline drift originates from the head or body movement

176 and is characterized by low-frequency electro-potential shift. White Gaussian noise was used to

177 represent the measurement noise.

178 Generally, about 15-20 eye blinks, each lasting 0.3-0.4 s will be done in $60 \mathrm{~s}$ when the 179 subject is relaxed. Based on the characters, a triangular pulse with the period of $3 \mathrm{~s}$ and the pulse width of $0.3 \mathrm{~s}$ was chosen to simulate the EOG artifact. A sinusoidal function with amplitude of $20 \mu \mathrm{V}$ and frequency of $0.5 \mathrm{~Hz}$ was used to simulate baseline drift.

Table 1. Parameters of the simulated EEG time series

\begin{tabular}{|c|c|c|c|}
\hline & Symbol & Value & Comments \\
\hline \multirow{12}{*}{ Spontaneous EEG } & $m_{1}(\mathrm{~Hz})$ & 3.61 & \multirow{3}{*}{ Delta rhythm } \\
\hline & $\sigma_{1}^{\xi}$ & 3.86 & \\
\hline & $\gamma_{1}$ & 0.97 & \\
\hline & $m_{2}(\mathrm{~Hz})$ & 5.76 & \multirow{3}{*}{ Theta rhythm } \\
\hline & $\sigma_{2}^{\xi}$ & 1.23 & \\
\hline & $\gamma_{2}$ & 0.99 & \\
\hline & $m_{3}(\mathrm{~Hz})$ & 10.45 & \multirow{3}{*}{ Alpha rhythm } \\
\hline & $\sigma_{2}^{\xi}$ & 1.57 & \\
\hline & $\gamma_{3}$ & 0.99 & \\
\hline & $m_{4}(\mathrm{~Hz})$ & 16.02 & \multirow{3}{*}{ Beta rhythm } \\
\hline & $\sigma_{4}^{\xi}$ & 0.92 & \\
\hline & $\gamma_{4}$ & 0.98 & \\
\hline \multirow{3}{*}{ Artifacts } & $V_{E O G}(\mu \mathrm{V})$ & $400 / 100$ & Amplitude of EOG \\
\hline & $T_{E O G}(\mathrm{sec})$ & 3 & Period of EOG \\
\hline & $P W_{E O G}(\mathrm{sec})$ & 0.3 & Pulse width of EOG \\
\hline
\end{tabular}




\begin{tabular}{|l|l|l|l|}
\hline & $V_{B L}(\mu \mathrm{V})$ & 20 & Amplitude of baseline drift \\
\cline { 2 - 4 } & $f_{B L}(\mathrm{~Hz})$ & 0.5 & Frequency of baseline drift \\
\hline
\end{tabular}

Parameters used in the EEG simulation are shown in Table 1. The data simulation and

treatment were carried out using MATLAB version 7.9.0 on a personal computer with Intel(R)

Core(TM) i5-4590 processor, 8 GB RAM and Windows 7 operating system.

\section{Experimental EEG data}

187

188

189

190

191

192

193

194

195

196

197

198

The experiments were approved with a protocol (NO. 20170010) by the Institutional Review Board of Tsinghua University. One male and two female aged 20-25 years participated in the experiments. These subjects were required to abstain from psychoactive substances for at least 4 h prior to experiments. Experiments were carried out with the subject sit on a comfortable chair in a room with normal lightness. The MP36 data acquisition and analysis system (BIOPAC Systems, Inc. USA) with a three electrodes system was used to acquire the EEG data. The $\mathrm{Ag} / \mathrm{AgCl}$ electrode (Wuxi Sichiray Technology Co. Ltd, China) flushed with conductive gel was attached to the scalp over the frontal region as the recording electrode. The other two electrodes were attached to the earlobe and mastoid, serving as a ground and a reference, respectively.

The experimental procedures were as follows. Initially, the subject closed eyes in relaxed state for $10 \mathrm{~min}$. After that, the subject opened eyes and visually fixated on a small cross displayed on a computer screen in front of him/her. Meanwhile, the EEG data started to be recorded with the sampling rate of $200 \mathrm{~Hz}$. Informed by the recorder, the subject then began 4 times of alternating periods of $60 \mathrm{~s}$ eyes open followed by $60 \mathrm{~s}$ eyes closed. The desired EEG segments were cut off from every period of the eyes-open and eyes-closed states. As shown in 
202

203

204

205

206

207

208

209

210

211

212

213

214

215

216

217

218

Fig. 3, each segment last for $8 \mathrm{~s}$ and was in the middle of each period. For each subject, the experiment was repeated 3 times in three separate days. Totally, 24 segments were obtained for each subject.

\section{Results and discussion}

\subsection{Artifacts removal}

\section{Simulated EEG data}

Simulated EEG data based on the MPA EEG model was used to verify the artifacts removal using the adaptive SSA. Representative results are shown in Fig. 4. When the EEG was contaminated with large artifacts (the EOG amplitude was $400 \mu \mathrm{V}$ ), as shown in Fig. 4A, the amplitude of the EEG signal was higher than the threshold $\left(V_{0}=200 \mu \mathrm{V}\right)$. The first two RCs were grouped as artifacts and removed. The corrected EEG and removed artifacts are shown in Fig. 4B and 4C, respectively. It can be seen that the amplitude of the corrected EEG was $54 \mu \mathrm{V}$, close to that of the simulated spontaneous EEG as shown in Fig. 2C. When the EEG was 
219

220

221

222 small artifacts could be removed adaptively by the adaptive SSA.

A)

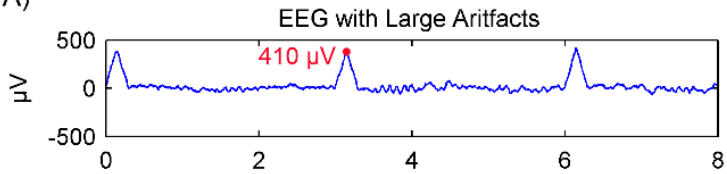

B)

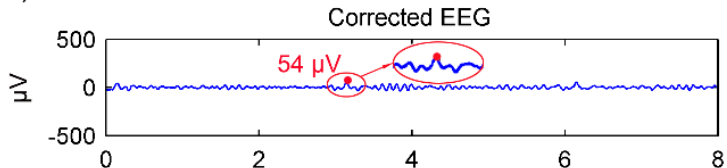

C)

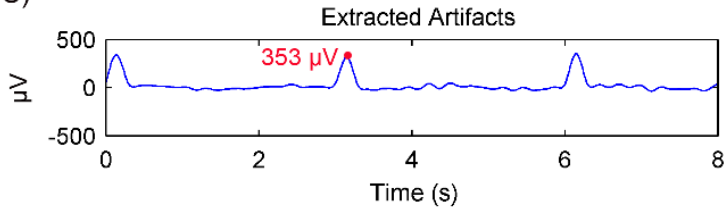

D)

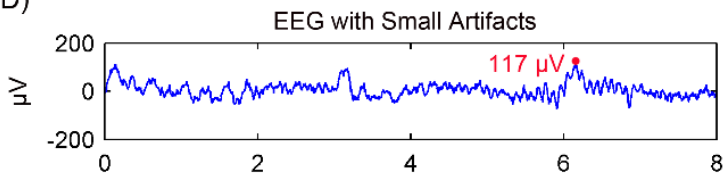

E)

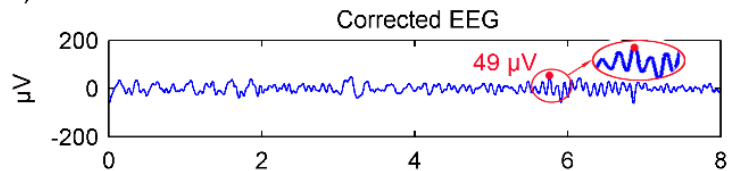

F)

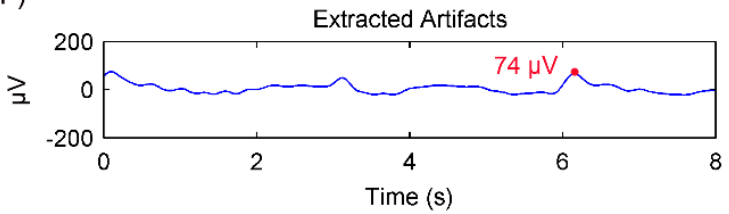

223

224

225

226

227

228

229

230

231

232

233

Fig. 4 Results of artifacts removal from simulated EEG using the adaptive SSA. EEG contaminated with artifacts (A), the corrected EEG (B) and removed artifacts (C), in the case of large artifacts. The amplitude of the EOG artifact is $400 \mu \mathrm{V}$. EEG contaminated with artifacts (D), the corrected EEG (E) and removed artifacts (F), in the case of small artifacts. The amplitude of the EOG artifact is $100 \mu \mathrm{V}$.

\section{Experimental EEG data}

The artifacts removal using the adaptive SSA was then further tested on experimental EEG data. Another recently reported SSA method (Maddirala \& Shaik 2016b), which will be called SSA 1\# in this paper, was used for comparison. The SSA 1\# used a novel grouping criterion based on the eigenvectors' local mobility, which is a signal complexity measure, to remove motion artifacts. 
234 The comparison results are shown in Fig. 5. For 9 of the total 24 segments, the corrected EEG

235 using the adaptive SSA was the same as that using the SSA 1\#. Representative results are shown

A)

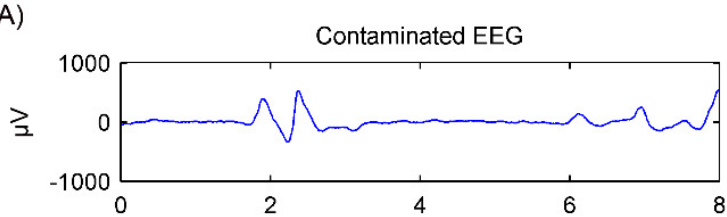

B)

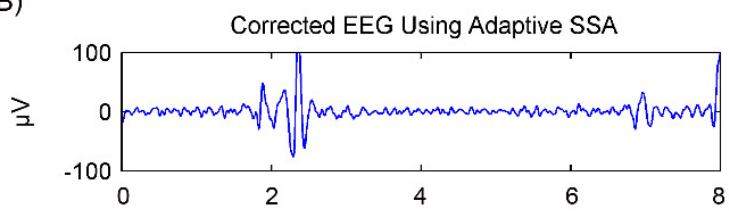

C)

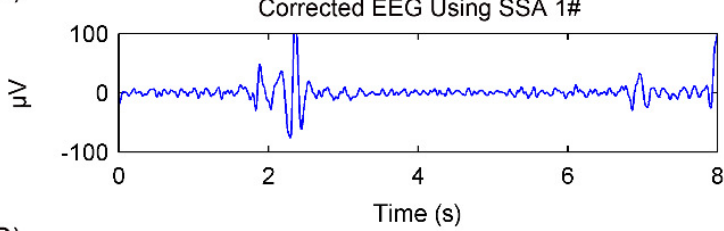

D)

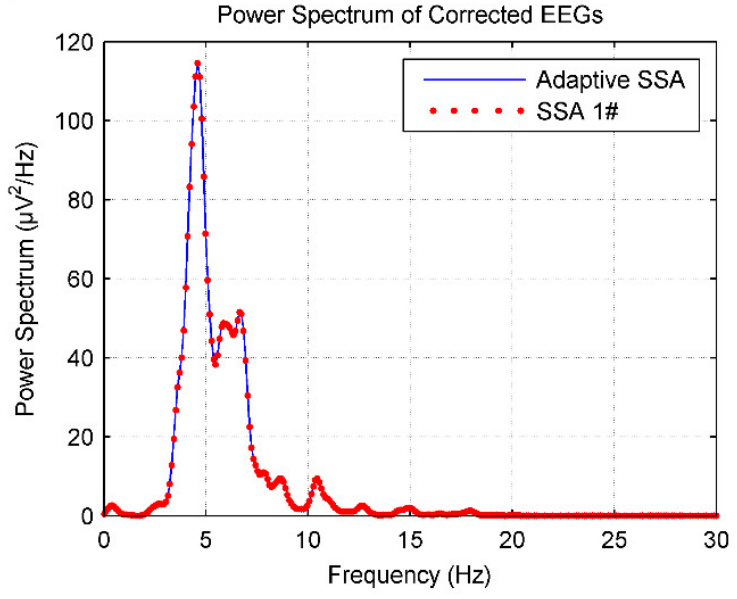

E)

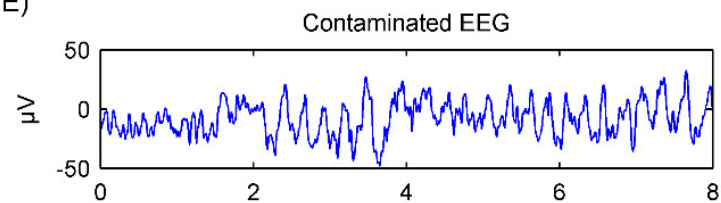

F)

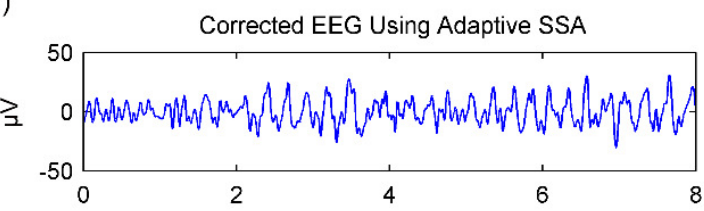

G)

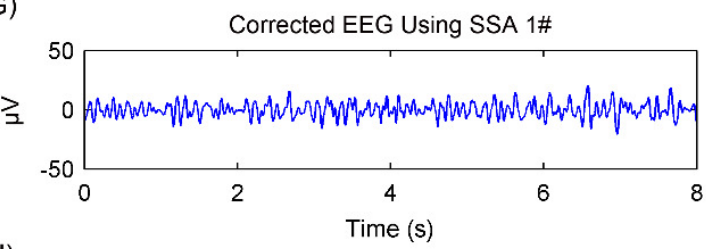

H)

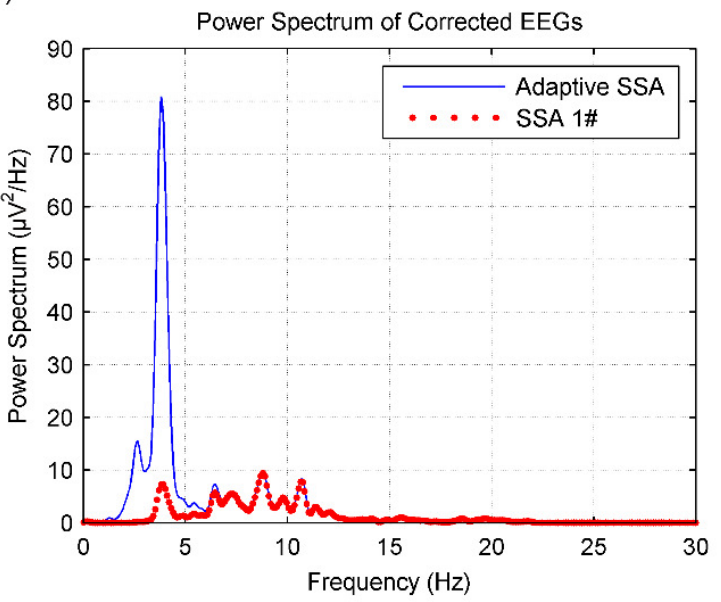

236

237 238

239

240

241

242 results using the adaptive SSA and SSA $1 \#$. 
243 in Fig. 5B and 5C. Furthermore, it can be seen from Fig. 5D that their power spectrums

244 overlapped completely. For the other 15 of the total 24 segments, the amplitude of the corrected

245 EEG using the adaptive SSA was higher than that of the corrected EEG using the SSA 1\#, as

246 shown in Fig. 5F and 5G, respectively. It can be seen from Fig. 5H that the SSA $1 \#$ removed

247 more artifacts than the adaptive SSA. The excess removed artifacts were in the frequency of 3-5

$248 \mathrm{~Hz}$, which was within the EEG frequency band and should not be removed. So, the corrected

249 EEG using the adaptive SSA was more complete and accurate than that using the SSA 1\#. It

250 verifies that the adaptive SSA has a similar, even better, artifacts removal effect.

\section{$251 \quad 3.2$ Alpha rhythms extraction}

\section{Simulated EEG data}

253 Simulated EEG data based on the MPA model was then used to verify the rhythms extraction

254 using the adaptive SSA. Results are shown in Fig. 6. The extracted alpha rhythm using the 255 adaptive SSA is shown in Fig. 6A. It was similar with the simulated alpha rhythm (shown in Fig.

256 6B). To further examine the rhythms extraction, their power spectrums were calculated (shown

257 in Fig. 6C). The power spectrum of the extracted alpha rhythm was in good consistence with that 258 of the simulated alpha rhythm. It verifies the validity of the adaptive SSA in rhythms extraction. 

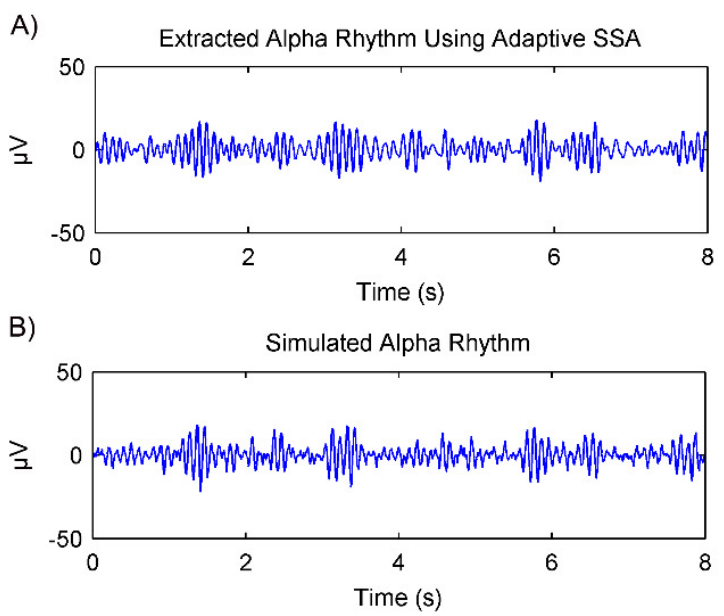

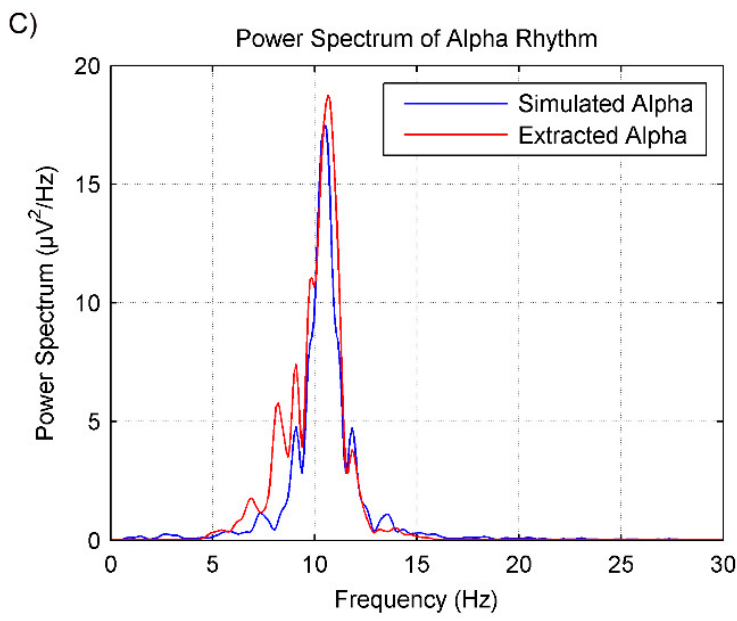

259

260

261

262

263

264

265

266

267

268

269

270

271

272

Fig. 6 Results of the alpha rhythm extraction from simulated EEG data using the adaptive SSA.

(A) The extracted alpha rhythm using the adaptive SSA. (B) The simulated alpha rhythm. (C)

Power spectrums of the alpha rhythms in (A) and (B).

\section{Experimental EEG data}

The rhythms extraction using the adaptive SSA was then further tested on experimental EEG data. After artifacts removal, the EEG alpha rhythms in the eyes-open and eyes-closed states were extracted. Fig. 7A and 7B shows the representative alpha rhythm extracted from EEG signals in the eyes-open and eyes-closed states, respectively. It is clear that the alpha rhythm in the eyes-open state was weaker than that in the eyes-closed state. Actually, the power value of the extracted alpha rhythm in the eyes-open state was $9.84 \mu \mathrm{V}^{2}$; while in the eyes-closed state the power value was $50.52 \mu \mathrm{V}^{2}$. Fig. 7C illustrates the alpha rhythm spectrogram, which is the square of the rhythm amplitude as a function of frequency. It presents an obvious difference between the eyes-open and eyes-closed states. 
A)

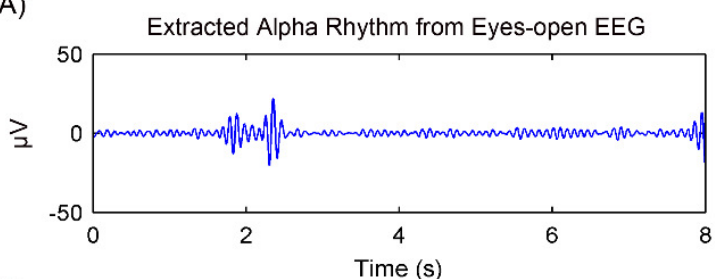

B)

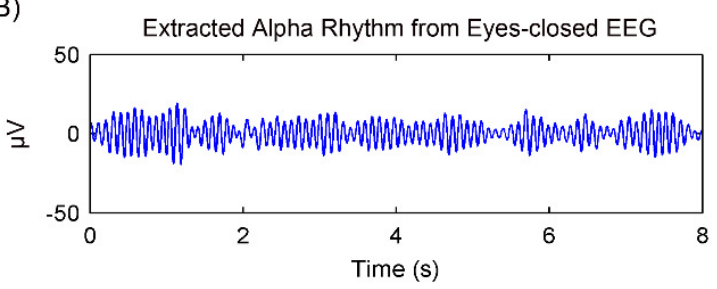

C)

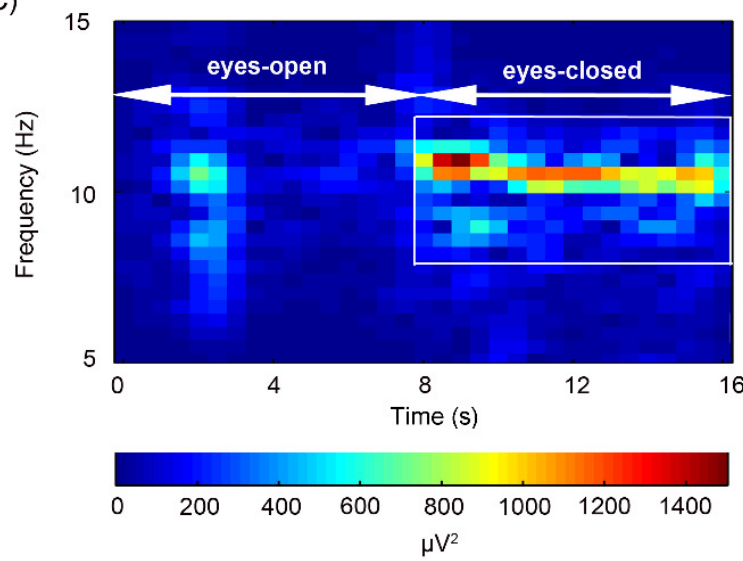

273

274

275

276

277

278

279

280

281

282

283

284

285

286

287

288

Fig. 7 Results of the alpha rhythm extraction in (A) eyes-open state and (B) eyes-closed state, respectively. (C) The spectrogram of the alpha rhythm.

In order to verify the rhythms extraction using the adaptive SSA, another recently reported SSA method (Mohammadi et al. 2016) and wavelet decomposition method (Akar et al. 2015), which will be called SSA 2\# and WDec respectively in this paper, were used for comparison. The comparison results are shown in Fig. 8. It can be seen from Fig. 8A and 8D that, in the eyes-open state, the extracted alpha rhythm using the adaptive SSA was of low amplitude and within the alpha band $(8-13 \mathrm{~Hz})$. So, it could represent the real alpha rhythm of EEG. From Fig. $8 \mathrm{~B}$ and $8 \mathrm{D}$, it can be seen that the SSA $2 \#$ could not extract any alpha rhythm in the eyes-open state. It was because the SSA 2\# could only extract the dominate component from EEG; while the alpha rhythm in the eyes-open state is weak and not the dominate component. Fig. 8C and 7D both show that the amplitude of the extracted alpha rhythm using the WDec in the eyesopen state was even higher than that in the eyes-closed state. It was obviously inconsistent with reality. Besides, it contained a large number of components out of the alpha band. Therefore, in the eyes-open state, the adaptive SSA performed better than both the SSA 2\# and WDec in 
289

290

291

292

293

294

A)

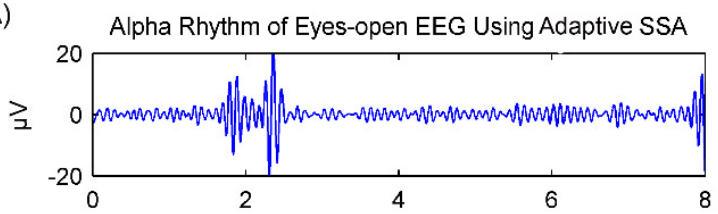

B)

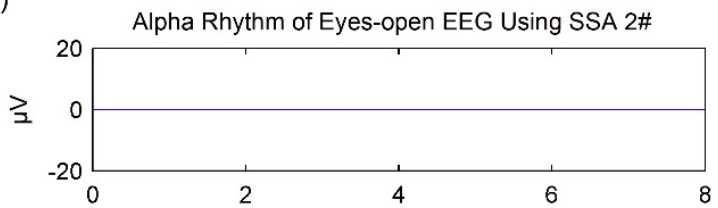

C)

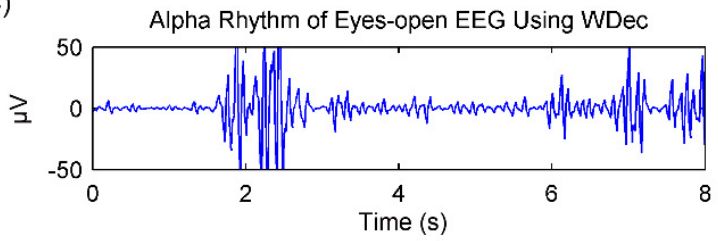

D)

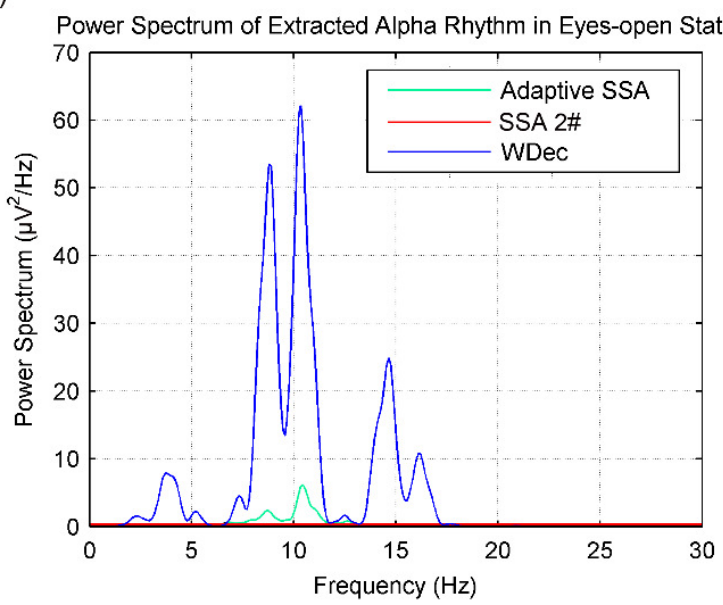

E)

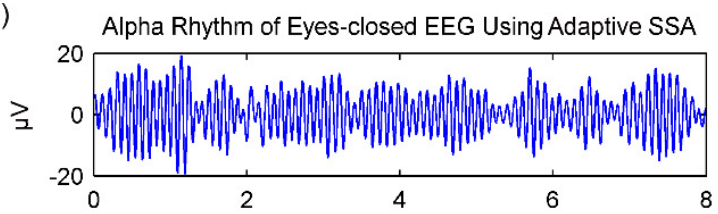

F)

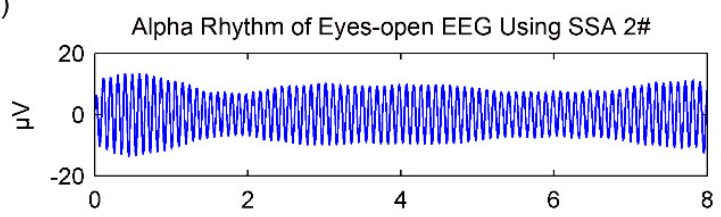

G)

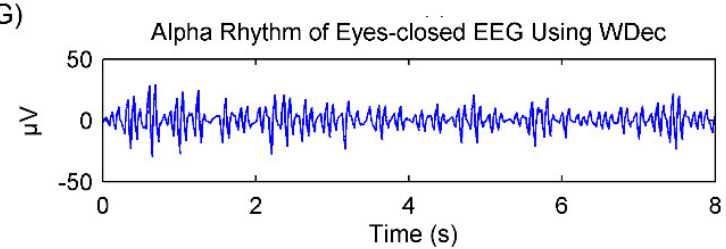

H)

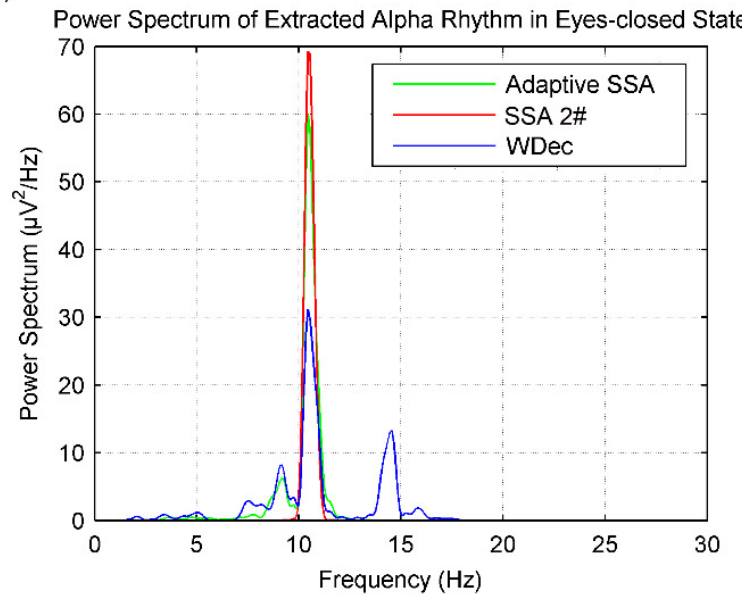

Fig. 8 Results of alpha rhythm extraction in the eyes-open and eyes-closed states using three 
298 and the WDec (C), and power spectrums of the extracted alpha rhythms (D), in the eyes-open

299 state. Extracted alpha rhythm using the proposed adaptive SSA (E), the SSA 2\# (F) and the

300 WDec $(\mathrm{G})$, and power spectrums of the extracted alpha rhythms $(\mathrm{H})$, in the eyes-closed state.

301

302

303

304

305

306

307

308

309

310

311

312

313

314

315

316

317

\subsection{Distinguishment between the eyes-open and eyes-closed states}

Previous studies have reported that the alpha rhythm in resting state with eyes closed is much stronger than that in the eyes-closed state with visual stimulation (Barry et al. 2007; Norton et al. 2015). Therefore, based on the alpha rhythm extraction, the adaptive SSA could be used to distinguish between the eyes-open and eyes-closed states. The extracted EEG alpha rhythms using the adaptive SSA are shown in Fig. 9A. In order to quantify the extracted alpha rhythms, their power values were calculated and shown in Fig. 9B. It is obvious that the power values in the eyes-open state were generally lower than those in the eyes-closed state. With a threshold power value of $10 \mu \mathrm{V}^{2}$, the two states could be distinguished. When the power was lower than the threshold, it was categorized as the eyes-open state. Otherwise, it was categorized as the eyes-closed state. The accuracy of the states distinguishment was $95.8 \%$.

The performance of the adaptive SSA was compared with the WDec and the infinite impulse response (IIR) filtering methods. Fig. 9C and 9D shows power values of the extracted alpha rhythms using the WDec and IIR filtering, respectively. It can be seen that, power values in the eyes-open state were generally lower than those in the eyes-closed state, similar to the results of the adaptive SSA. However, the power values obtained using the WDec and IIR filtering were larger than those using the adaptive SSA. It was likely because the WDec and IIR 
318 filtering could not remove the artifacts within the alpha band. With a threshold power value of

$31920 \mu \mathrm{V}^{2}$, the WDec and IIR filtering could both achieve the optimal distinguishment. The

320 accuracy of states distinguishment was $79.2 \%$ and $83.3 \%$, respectively, lower than the adaptive

321 SSA. It can be concluded that the adaptive SSA could be potentially used to distinguish between

322 the eyes-open and eyes-closed states.

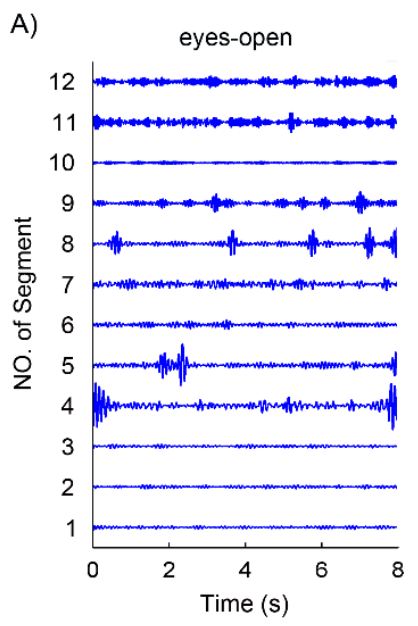

C)
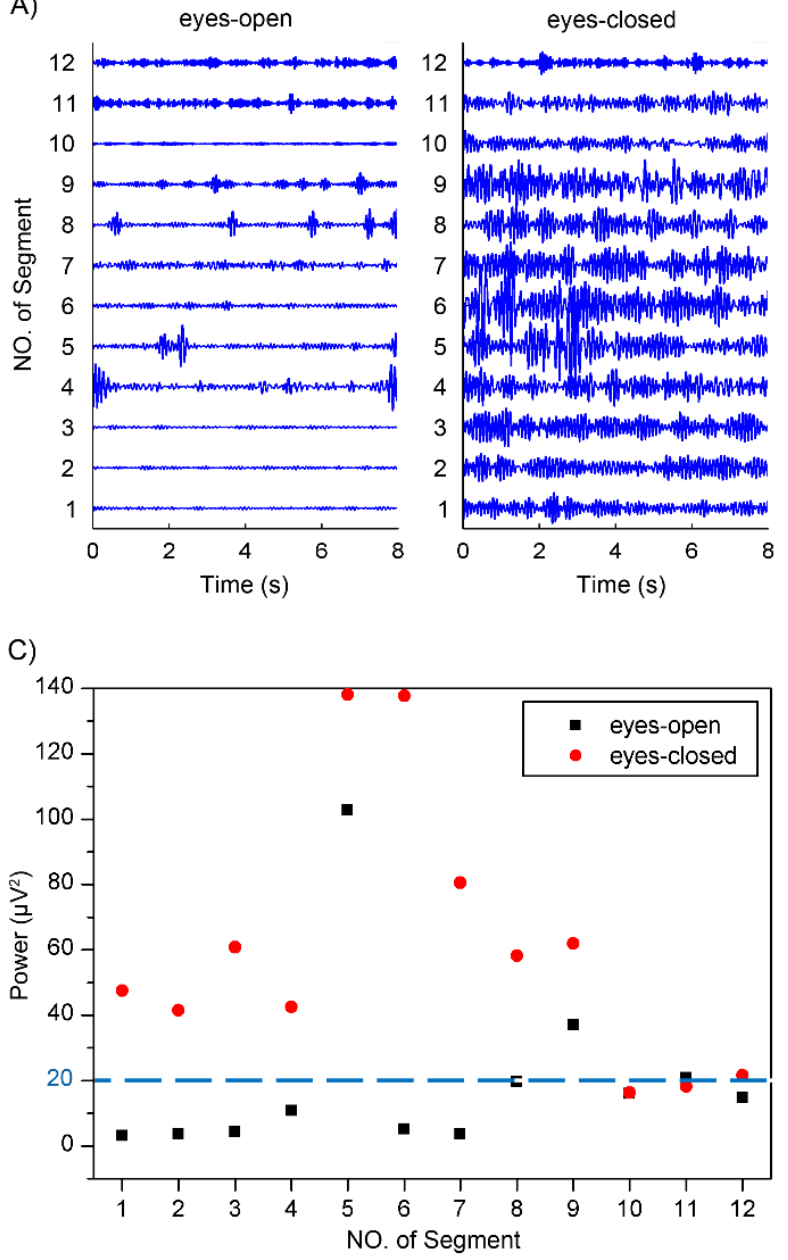

B)

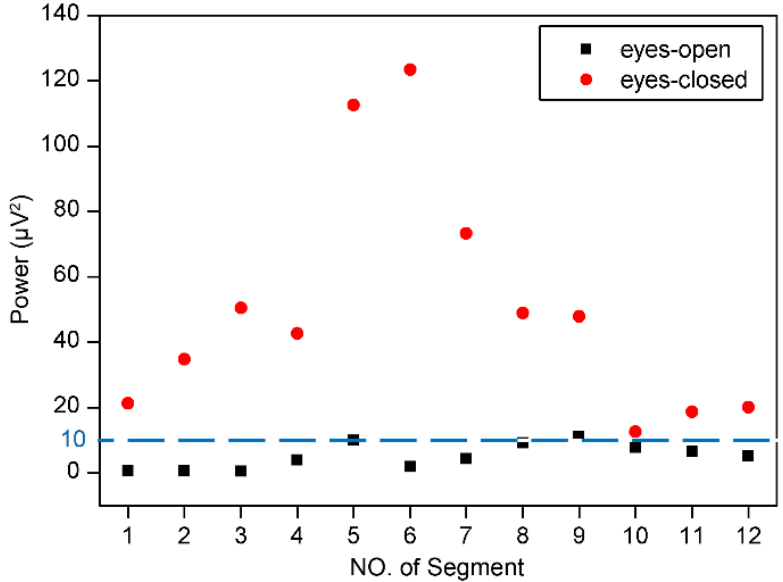

D)

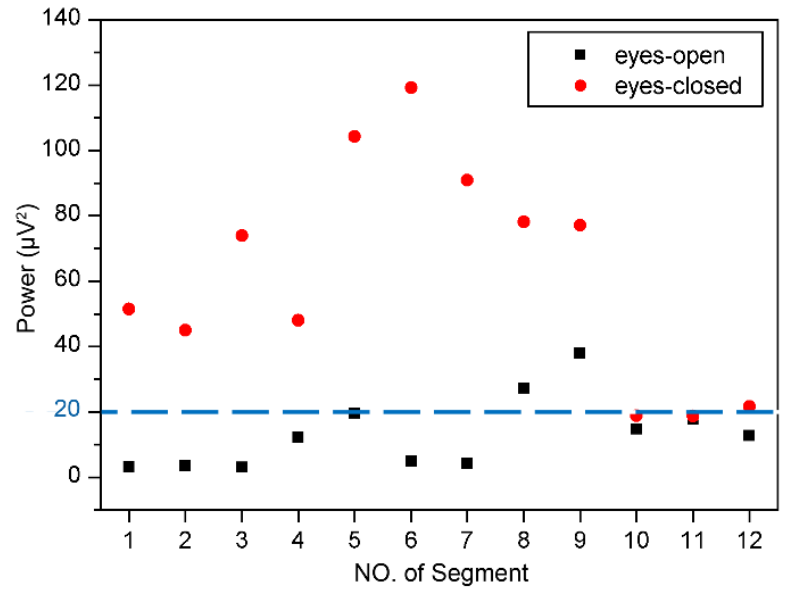

Fig. 9 (A) Extracted alpha rhythms using the adaptive SSA. (B) Power values of alpha rhythms using the adaptive SSA. (C) Power values of alpha rhythms using the WDec. (D) Power values of alpha rhythms using the IIR filtering. 
327

328

329

330

331

332

333

334

335

336

337 338

339

340

341

342

343

\section{Conclusions}

In this paper, we proposed an adaptive SSA method with a novel grouping rule to remove artifacts and extract alpha rhythms from EEG signals in eyes-open and eyes-closed states. The grouping rule enables SSA to be adaptive to EEG signals containing different levels of artifacts and rhythms. In order to verify the validity of the proposed adaptive SSA, the simulated EEG data based on the Markov Process Amplitude (MPA) EEG model and the experimental EEG data in eyes-open and eyes-closed states were used. The proposed adaptive SSA showed a better performance in artifacts removal and rhythms extraction, than another two recently reported SSA methods and the WDec method. Additionally, a proof-of-concept experiment was performed to apply the adaptive SSA to distinguish between the eyes-open and eyes-closed states. Results showed an accuracy of $95.8 \%$, higher than that of the WDec method $(79.2 \%)$ and the IIR filtering method $(83.3 \%)$.

\section{Appendix}

\section{Markov Process Amplitude (MPA) EEG model}

As described in section 3.1, the EEG is a combination of several oscillations. The MPA EEG model can be constructed as

$$
\left\{\begin{array}{c}
s(n \Delta t)=\sum_{i=1}^{K} a_{i}(n \Delta t) \sin \left(2 \pi m_{i} n \Delta t+\theta_{i}\right) \\
a_{i}[(n+1) \Delta t]=\gamma_{i} a_{i}(n \Delta t)+\xi_{i}(n \Delta t) \\
0<\gamma_{i}<1 \quad i=1,2, \mathrm{~K}, K
\end{array}\right.
$$


344 The theoretical power spectrum of the MPA EEG model was given as

$$
P(f)=\sum_{i=1}^{K}\left\{\frac{0.25 \Delta t\left(\sigma_{i}^{\xi}\right)^{2}}{1+\left(\gamma_{i}\right)^{2}-2 \gamma_{i} \cos \left[2 \pi \Delta t\left(f-m_{i}\right)\right]}+\frac{0.25 \Delta t\left(\sigma_{i}^{\xi}\right)^{2}}{1+\left(\gamma_{i}\right)^{2}-2 \gamma_{i} \cos \left[2 \pi \Delta t\left(f+m_{i}\right)\right]}\right\}
$$

346

347 348

350

351

352

353

354

355

356

357

358

359

360

361

362

363

364

In the power spectrum of the EEG, the width and the amplitude of peak frequency are the most important features. Define $H_{i}$ as the amplitude and the $F_{i}$ as frequency width at half of $H_{i} . H_{i}, F_{i}$ can be described as

$$
\begin{gathered}
H_{i}=\frac{\Delta t\left(\sigma_{i}^{\xi}\right)^{2}}{4\left(1-\gamma_{i}\right)^{2}} \\
F_{i}=\frac{1}{\pi \Delta t} \cos ^{-1} \frac{4 \gamma_{i}-1-\left(\gamma_{i}\right)^{2}}{2 \gamma_{i}}
\end{gathered}
$$

In order to determine parameters of the MPA EEG model, the power spectrum of the real EEG was calculated using the Welch method. The amplitude and width of peak frequency $\left(m_{i}\right)$ in deferent oscillations (delta, theta, alpha and beta) were obtained as $H_{i}$ and $F_{i}$. Then the parameters of the model, $\gamma_{i}$ and $\sigma_{i}^{\xi}$, were determined.

\section{References}

Akar SA, Kara S, Latifoğlu F, and Bilgic V. 2015. Investigation of the noise effect on fractal dimension of EEG in schizophrenia patients using wavelet and SSA-based approaches. Biomedical Signal Processing and Control 18:42-48.

Al-Nashash H, Al-Assaf Y, Paul J, and Thakor N. 2004. EEG signal modeling using adaptive Markov process amplitude. IEEE transactions on biomedical engineering 51:744-751.

Azami H, and Sanei S. 2012. Automatic signal segmentation based on singular spectrum analysis and imperialist competitive algorithm. International Econference on Computer and Knowledge Engineering. p 50-55.

Azami H, and Sanei S. 2014. Spike detection approaches for noisy neuronal data: Assessment and comparison. Neurocomputing 133:491-506. 
365

366

367

368

369

370

371

372

373

374

375

376

377

378

379

380

381

382

383

384

385

386

387

388

389

390

391

392

393

394

395

396

397

398

399

400

401

402

403

404

405

Azarbad M, Azami H, Sanei S, and Ebrahimzadeh A. 2014. A Time-frequency Approach for EEG Signal Segmentation. Ultrasound in Obstetrics \& Gynecology 22:180-182.

Bai O, Nakamura M, Ikeda A, and Shibasaki H. 2000. Nonlinear Markov process amplitude EEG model for nonlinear coupling interaction of spontaneous EEG. IEEE transactions on biomedical engineering 47:1141-1146.

Bai O, Nakamura M, Nishida S, Ikeda A, and Shibasaki H. 2001. Markov process amplitude EEG model for spontaneous background activity. Journal of Clinical Neurophysiology 18:283-290.

Barry RJ, Clarke AR, Johnstone SJ, Magee CA, and Rushby JA. 2007. EEG differences between eyes-closed and eyesopen resting conditions. Clinical Neurophysiology 118:2765-2773.

Chi YM, and Cauwenberghs G. 2010. Wireless Non-contact EEG/ECG Electrodes for Body Sensor Networks. International Conference on Body Sensor Networks. p 297-301.

Daly I, Nicolaou N, Nasuto SJ, and Warwick K. 2013. Automated artifact removal from the electroencephalogram: a comparative study. Clinical EEG and Neuroscience 44:291-306.

Gargiulo G, Bifulco P, Calvo RA, Cesarelli M, Jin C, and van Schaik A. 2008. A mobile EEG system with dry electrodes. Biomedical Circuits and Systems Conference, 2008 BioCAS 2008 IEEE: IEEE. p 273-276.

He DP, Wilson G, and Russell C. 2004. Removal of ocular artifacts from electro-encephalogram by adaptive filtering. Medical \& Biological Engineering \& Computing 42:407-412.

James CJ, and Lowe D. 2003. Extracting multisource brain activity from a single electromagnetic channel. Artificial Intelligence in Medicine 28:89-104.

Jones SG, Riedner BA, Smith RF, Ferrarelli F, Tononi G, Davidson RJ, and Benca RM. 2014. Regional reductions in sleep electroencephalography power in obstructive sleep apnea: a high-density EEG study. Sleep 37:399407.

Ko K-E, Yang H-C, and Sim K-B. 2009. Emotion recognition using EEG signals with relative power values and Bayesian network. International Journal of Control, Automation and Systems 7:865.

Kouchaki S. 2014. An Adaptive Filtering Approach Using Supervised SSA for Identification of Sleep Stages From EEG. Frontiers in Biomedical Technologies 1.

Maddirala AK, and Shaik RA. 2016a. Motion artifact removal from single channel electroencephalogram signals using singular spectrum analysis. Biomedical Signal Processing and Control 30:79-85.

Maddirala AK, and Shaik RA. 2016b. Removal of EOG Artifacts from single channel EEG signals using combined singular spectrum analysis and adaptive noise canceler. IEEE Sensors Journal 16:8279-8287.

Mees A, Rapp P, and Jennings L. 1987. Singular-value decomposition and embedding dimension. Physical Review $A$ 36:340.

Mohammadi SM, Enshaeifar S, Ghavami M, and Sanei S. 2015. Classification of awake, REM, and NREM from EEG via singular spectrum analysis. Engineering in Medicine and Biology Society. p 4769-4772.

Mohammadi SM, Kouchaki S, Ghavami M, and Sanei S. 2016. Improving time-frequency domain sleep EEG classification via singular spectrum analysis. Journal of Neuroscience Methods 273:96-106.

$\mathrm{Ng} \mathrm{KA}$, and Chan PK. 2005a. A CMOS analog front-end IC for portable EEG/ECG monitoring applications. Circuits and Systems I: Regular Papers, IEEE Transactions on 52:2335-2347.

$\mathrm{Ng} \mathrm{KA}$, and Chan PK. 2005b. A CMOS analog front-end IC for portable EEG/ECG monitoring applications. IEEE Transactions on Circuits \& Systems I Regular Papers 52:2335-2347.

Niedermeyer E, and da Silva FL. 2005. Electroencephalography: basic principles, clinical applications, and related fields: Lippincott Williams \& Wilkins. 
406

407

408

409

410

411

412

413

414

415

416

417

418

419

420

421

422

423

424

425

426

427

428

429

430

431

432

433
Nishida S, Nakamura M, and Shibasaki H. 1986. An EEG model expressed by sinusoidal waves with the Markov process amplitude. Iyo denshi to seitai kogaku Japanese journal of medical electronics and biological engineering 24:8-14.

Norton JJ, Lee DS, Lee JW, Lee W, Kwon O, Won P, Jung S-Y, Cheng H, Jeong J-W, and Akce A. 2015. Soft, curved electrode systems capable of integration on the auricle as a persistent brain-computer interface. Proceedings of the National Academy of Sciences 112:3920-3925.

Nunez PL, Silberstein RB, Shi Z, Carpenter MR, Srinivasan R, Tucker DM, Doran SM, Cadusch PJ, and Wijesinghe RS. 1999. EEG coherency II: experimental comparisons of multiple measures. Clinical Neurophysiology 110:469-486.

Rowland N, Meile M, and Nicolaidis S. 1985. EEG alpha activity reflects attentional demands, and beta activity reflects emotional and cognitive processes. Science 228:750-752.

Sanei S, Ghodsi M, and Hassani H. 2011. An adaptive singular spectrum analysis approach to murmur detection from heart sounds. Medical Engineering \& Physics 33:362-367.

Sanei S, Lee TKM, and Abolghasemi V. 2012. A New Adaptive Line Enhancer Based on Singular Spectrum Analysis. IEEE transactions on bio-medical engineering 59:428.

Takens F. 1981. Detecting strange attractors in turbulence. Dynamical systems and turbulence, Warwick 1980: Springer, 366-381.

Teixeira AR, Tomé AM, Lang EW, Gruber P, and Martins dSA. 2006. Automatic removal of high-amplitude artefacts from single-channel electroencephalograms. Computer Methods \& Programs in Biomedicine 83:125-138.

Thuraisingham RA, Tran Y, Boord P, and Craig A. 2007. Analysis of eyes open, eye closed EEG signals using secondorder difference plot. Medical \& Biological Engineering \& Computing 45:1243-1249.

Van Bavel M, Leonov V, Yazicioglu RF, Torfs T, Van Hoof C, Posthuma N, and Vullers R. 2008. Wearable battery-free wireless 2-channel EEG systems powerd by energy scavengers. Sensors \& Transducers Journal 94:103-115.

Vautard R, Yiou P, and Ghil M. 1992. Singular-spectrum analysis: A toolkit for short, noisy chaotic signals. Physica D: Nonlinear Phenomena 58:95-126.

Wallstrom GL, Kass RE, Miller A, Cohn JF, and Fox NA. 2004. Automatic correction of ocular artifacts in the EEG: a comparison of regression-based and component-based methods $\bar{r}$. International Journal of Psychophysiology 53:105-119. 